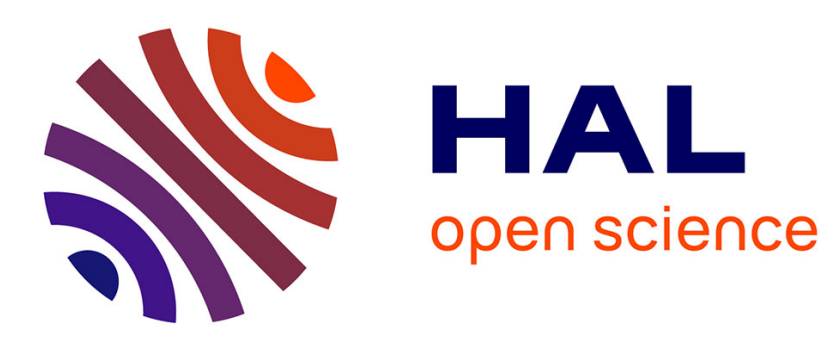

\title{
Fishing for excuses and performance evaluation
}

François Larmande, Jean-Pierre Ponssard

\section{To cite this version:}

François Larmande, Jean-Pierre Ponssard. Fishing for excuses and performance evaluation. 2013. hal-00825297

\section{HAL Id: hal-00825297 \\ https://hal.science/hal-00825297}

Preprint submitted on 23 May 2013

HAL is a multi-disciplinary open access archive for the deposit and dissemination of scientific research documents, whether they are published or not. The documents may come from teaching and research institutions in France or abroad, or from public or private research centers.
L'archive ouverte pluridisciplinaire HAL, est destinée au dépôt et à la diffusion de documents scientifiques de niveau recherche, publiés ou non, émanant des établissements d'enseignement et de recherche français ou étrangers, des laboratoires publics ou privés. 


\section{ECOLE POLYTECHNIQUE}

CENTRE NATIONAL DE LA RECHERCHE SCIENTIFIQUE

FISHING FOR EXCUSES AND PERFORMANCE EVALUATION

François LARMANDE

Jean-Pierre PONSSARD

Cahier n 2013-11

\section{DEPARTEMENT D'ECONOMIE}

Route de Saclay

91128 PALAISEAU CEDEX

(33) 169333033

http://www.economie.polytechnique.edu/

mailto:chantal.poujouly@polytechnique.edu 


\title{
Fishing for excuses and performance evaluation
}

\author{
F. Larmande* $\quad$ J.P. Ponssard ${ }^{\dagger}$
}

May 2013

\begin{abstract}
We study a principal-agent model in which the agent can provide ex post additional relevant information regarding his performance. In particular, he can provide a legitimate excuse, that is, evidence that a poor result is only due to factors outside his control. However, building a convincing case requires time, time that is not spent on exerting productive effort, and thus generating information represents an opportunity cost. We obtain necessary and sufficient conditions for the principal to prefer a policy of adjusting ex post the performance measure for the information provided by the agent to a policy of conforming to a result-based system with no adjustments. The risk aversion and a possible limited liability of the agent play an important role in the analysis. This paper clarifies the issues associated with the so-called "excuse culture" prevailing in some organizations.
\end{abstract}

JEL Classification D82 - M41 · M52

Keywords Performance measurement · Manipulation · Controllability principle $\cdot$ Excuse culture $\cdot$ Influence activity

*EMLYON, France. Email: larmande@em-lyon.com (corresponding author).

${ }^{\dagger} \mathrm{CNRS}$ and Ecole polytechnique, Palaiseau, France. 


\section{Introduction}

"In an excuse culture, instead of focusing on generating good results and being committed to achieving their targets, employees spend considerable amounts of time making excuses and lobbying their evaluators (...). And the negotiation and, potentially, appeal process distract employees from the real tasks at hand." Merchant and Van der Stede (2003) p. 470.

Evaluation schemes in practice swing between two extremes: resultbased with no adjustments or result-based with adjustments for the impact of uncontrollable factors. The latter approach might be beneficial in reducing the risk borne by the agent; but agents, by providing the relevant information, are often themselves at the origin of the adjustment, which may lead to an undesirable "culture of excuses" characterized by agents who spend too much time on unproductive influence activities (Milgrom and Roberts 1988) rather than on productive tasks.

In this contribution, we introduce into a standard principal-agent model the idea that the agent can provide ex post relevant information regarding his performance. In particular, he can provide a legitimate excuse: evidence that a poor result is only due to factors outside his control. He may also provide hard information that a good result is not a windfall due to a favorable environment but is brought about by his own performance. However, generating relevant information requires time, time not spent on exerting productive effort. It represents an opportunity cost. We suppose that the principal has the opportunity to encourage or not the production of relevant information through the design of the incentive scheme.

The opportunity cost of generating information is a key parameter in the analysis. For the agent to be able to provide relevant information, especially a legitimate excuse, two conditions must be met. First, the true performance, once the effect of external factors has been filtered out, must be good. Second, the agent must be able to provide verifiable and convincing evidence to the principal. A productive effort, which is what the principal really wants to encourage, has two opposite effects: it increases the prob- 
ability of a good performance, but decreases the probability of providing verifiable information. The equilibrium between the two effects, and thus the degree of informativeness of this additional information, is a function of the opportunity cost of generating information.

In this analysis, we give due attention to the empirical fact that evaluators may not be able to condition rewards on whether or not an adjustment has been made. ${ }^{1}$ Such an impossibility to contract on the details of the production of the performance measure might arise if the task of verifying the information is performed by a third party, an auditor for instance. As argued by Arya and Glover (2008), managers routinely provide corrections that may be incorporated in the final audit. Conditioning pay on the extend to which the result has been adjusted, seems either prohibitively costly or unverifiable. Thus, only the final result is verifiable, but not all the details of its production.

Firstly, as a benchmark we assume that the principal is able to offer the most complete contract, i.e. to differentiate the incentive scheme on the provision of additional information. There are three possibilities. If the opportunity cost of providing information is high, a result-based system with no adjustments is optimal. For intermediate values, the principal rewards the provision of an excuse, but with a lower wage than the one resulting from an uncorrected good result. If the opportunity cost is sufficiently low, the principal again always rewards the provision of an excuse, but now with a higher wage than in a case of a good result without additional information. In this scheme, the principal induces the agent to provide further information even if the result is good: in order to obtain the highest possible reward, the agent has to prove that a high result is not a windfall. Although rare in practice, such situations may occur when the information directly available

\footnotetext{
${ }^{1}$ Larmande and Ponssard (2008) provide anecdotal evidence on how an appeal process might be implemented in practice. In this case study of the implementation of an EVA incentive scheme, introduced initially to foster a culture of results, the company did adjust the result when excuses were considered legitimate. For instance, by neutralizing the impact of a devaluation of local currency on the cost of invested capital, or the impact a regulatory change in the calculation of pension cost. While results were adjusted, the bonus function remained unchanged. Equity concerns were advanced to motivate such practices.
} 
to the principal is of poor informativeness, and much of the information is provided by the agent, information easily verifiable and not too costly to produce. As an illustration, the reward system of professors in universities comes to mind, wherein the information used for stewardship is the number of extra hours, of theses supervised, publications and editing activities information often directly provided by the professors themselves.

Secondly, we suppose that the principal is unable to contract on the fact that adjustments have been made or not. The same rewards must be offered whether or not the result has been adjusted. Note that the agent has no more incentive to prove that a good result is not a windfall - he already obtained the highest possible bonus. Thus, only excuses may be provided. The main change arises for intermediate values of the opportunity cost. The benefit of rewarding excuses is then to detect shirking more effectively in the case of a low outcome: the fact that the result is low and that the agent is unable to provide an excuse is very informative of shirking. The risk of punishing an agent who has made an effort is thus lower. The drawback of rewarding excuses is to increase the likelihood of a windfall: rewarding with a high wage an agent who has actually shirked. The optimal contract depends then on the risk aversion of the agent. With high risk aversion more weight is put on the former effect than on the latter, and allowing excuses is optimal. With low risk aversion, the reverse holds.

In practice, evaluators may also be constrained in the possible punishment they can impose in case of a poor result. In Section 3, we investigate the impact of the agent's potential limited liability. Not allowing adjustments is in this case more often optimal because of the rent the agent now earns, which represents another agency cost for the principal. For intermediate values of the opportunity cost, rewarding excuses leads to a higher rent, which can outweigh its risk-sharing advantage. Then, only if the agent's risk aversion is sufficiently high is the policy of rewarding excuses optimal. We show that this is the case even if the principal can differentiate the rewards when an adjustment takes place.

The remainder of the article is organized as follows. Section 2 reviews the literature directly related to the topic. Section 3 presents the model and 
gives a synthetic preview of the results. Sections 4,5 and 6 respectively characterize the optimal incentive scheme under the three possibilities we consider. Section 7 concludes. The proofs may be found in the appendix.

\section{Related literature}

Providing an excuse is an example of an "influence activity" (Milgrom and Roberts (1988)). That is, an action of lobbying the principal that is costly because the time spent doing it is not devoted to valuable productive activities, but which provides valuable information not directly available to the evaluator otherwise. Here, the information concerns the result of the agent's effort; whereas in Milgrom and Roberts, it relates to abilities for a higher position.

Manipulating the result through "windows dressing" is another example of an action that is unproductive - the true performance is not affected -, and may be costly due to the time it required. Yet, the principal could well be better off to let the agent undertake this unproductive action. Feltham and Xie (1994) argue that using a performance measure prone to costly manipulation such as accounting numbers might be optimal because the overall noise is lower than the noise of another measure (the share price) less easy to manipulate. Demski (1998) introduces the idea that the possibility of manipulation depends on the productive action itself. Dutta and Gigler (2002) and Demski et al. (2004) provide alternative justifications that are not based on a risk-sharing issue. In our work, the agent does not directly manipulate the measure but influences the information process used to determine the bonus. However, as in Demski, the likelihood of the provision of an excuse depends on the productive effort. Moreover, here the noise of the measure is not uniformly affected: if the opportunity cost is not too great, accepting excuses makes a poor result less noisy (more informative of shirking) - with the possible drawback of increasing the noise for a good outcome.

Another closely related paper is Arya and Glover (2008). They analyze a setting in which the agent can provide additional information ex post. As noted previously, they consider that the incentive scheme cannot be subject 
to the condition of whether or not additional information has been provided. In their model, the agent performs two tasks. They analyze whether the principal is better off to accept an excuse related to one task or to accept an excuse only when verifiable information for both tasks is provided. In our work, we compare an excuse contract with a no-excuse contract. They assume a risk-neutral agent, while we introduce risk aversion. We point out that a trade-off arises for intermediate values of the informativeness of an excuse: the likelihood ratio of a windfall is higher when excuses are allowed; whereas, the likelihood ratio of not rewarding an agent who has made a productive effort is lower. This trade-off does not appear when the agent is risk-neutral: only the former likelihood ratio matters.

\section{Model}

A principal (she) hires an agent (he) to work on a task during one period. The agent may either make a productive effort ( $a=e$ associated with a private cost $\left.C_{p}=C>0\right)$ or shirk $\left(a=0, C_{p}=0\right)$. The result of the task, denoted $y$, may either be high (good) or low (insufficient), denoted as $y^{H}$ and $y^{L}$ respectively, with $y^{H}>y^{L}$. Let $\pi(a)=\operatorname{Prob}\left(y^{H} \mid a\right)$ denote the probability of obtaining a high result, given the choice of action $a$. A productive effort increases the probability of obtaining a high result: $\pi(e)>$ $\pi(0)$.

Ex post, the agent can provide additional and verifiable information regarding his task. This additional information might be a legitimate excuse; that is, evidence that a poor result is due to an adverse environment and that if the effect of the environment is filtered out, the underlying performance is actually high. Or, it might be evidence that a high result is not a windfall, that is, only due to a favorable environment. However, the gathering of information is not free: building a convincing case takes time, time not spent on the productive task. Therefore, we assume that the probability, denoted $q(a)$, of generating verifiable information that may convince the principal is lower when the agent makes a productive effort: $q(e) \leq q(0)$. The lower $q(e)$ relative to $q(0)$, the larger the opportunity cost for generating 
verifiable information.

Let us provide now the information structure in more detail. See our Figure 1. We assume that the result $y$ is a garbling of the underlying performance $x$ that can be high: $x=x^{H}$, or low: $x=x^{L}$. The agent influences only the random variable $x$, not the garbling between $x$ and $y$. The principal does not observe the performance $x$, only the result $y$. Let $p(a)$ represent the probability of obtaining a high performance $x^{H}$ if the agent chooses action $a$. Let $n_{H}$ denote the probability of obtaining a high result, conditionally on the performance being equal to $x^{H}$, and $n_{L}$ denote the probability of obtaining a low result, conditionally on the performance being equal to $x^{L}$. We have therefore:

$$
\pi(a)=p(a) n_{H}+(1-p(a)) n_{L}
$$

It is assumed that $n_{H}>n_{L}$ and $p(e)>p(0)$.

The additional information the agent can provide relates to the decomposition of the result into controllable performance and external environment: with probability $q(a)$, he is able to provide this decomposition; that is, to prove the value of his performance $x$.

The principal is risk-neutral. The agent is risk-averse. The agent has a utility function increasing, concave and separable in wage and private cost, $u(w)-C_{P}$. The expected utility of the agent must at all times be higher than a given reservation utility $u_{0}$. Two cases will be further distinguished depending on whether the agent is or is not protected by a limited liability. The limited liability entails that wage $w$ must always be non-negative.

We assume that the principal always wants to implement action $e$. Moreover, we assume that she is able to commit to rewarding only verifiable information provided by the agent. We rule out any renegotiation after effort based on soft information as in Fudenberg and Tirole (1990). We want to know when it is optimal for the principal to design a contract in which the verifiable information provided by the agent is taken into account. 


\section{Likelihood ratios of an effort}

As usual in agency theory, the likelihood ratio of whether the agent did or did not make an effort plays a crucial role in the analysis. Denote this likelihood ratio $R^{H}$ when a high result is observed and $R^{L}$ when a low one is:

$$
R^{H}=\pi(e) / \pi(0)
$$

Similarly,

$$
R^{L}=(1-\pi(e)) /(1-\pi(0))
$$

We will say that the agent provides a (legitimate) excuse when the result is low and he provides evidence that his performance is high. Let $\epsilon$ represent this event. Denote $R^{\epsilon}$ the likelihood ratio of an effort when such an excuse is provided. Given the choice of action $a$, the probability that the agent provides an excuse is the probability that jointly the true performance is

high $\left(x=x^{H}\right)$, the uncorrected result is low $\left(y=y^{L}\right)$, and the excuse is provided; that is, $p(e) q(e)\left(1-n_{H}\right)$. Thus:

$$
\begin{aligned}
R^{\epsilon} & =p(e) q(e)\left(1-n_{H}\right) /\left(p(0) q(0)\left(1-n_{H}\right)\right) \\
& =p(e) q(e) /(p(0) q(0))
\end{aligned}
$$

We will say that the agent proves that a high result is not due to a windfall when he provides further evidence that his performance is high. Let $\eta$ represent this event. The probability of this event is $p(e) q(e) n_{H}$. The associated likelihood ratio $R^{\eta}$ is equal to $R^{\epsilon}$. In other words, when the agent provides additional information, we have the same likelihood ratio whether the result is high or low because the agents control only the performance and not the garbling between the performance and the result.

For notational simplicity, henceforth let $R^{\epsilon}$ denote either of the two likelihood ratios.

We have for certain $R^{H}>1>R^{L}$, while $R^{\epsilon}$ may be higher than $R^{H}$, 
lower than $R^{L}$ or anywhere in between, depending on the opportunity cost of providing an excuse, i.e. the ratio $q(e) / q(0)$. The higher the opportunity cost, the lower this ratio and the lower $R^{\epsilon}$. Intuitively, the higher the opportunity cost, the lower the incentive for the principal to allow the agent to provide an excuse. This intuition is globally correct in our setting, regardless of the precise characteristics of the contract. This feature is demonstrated in Figure 2 that presents a synthetic preview of our results. How the boundary evolves between allowing and not allowing for adjustments will now be discussed in detail. The role of risk aversion will also be analyzed.

For future convenience, we introduce the probability that the result is high or, if the result is low, that the agent provides an excuse. Denote $\varphi(a)$ this probability.

$$
\varphi(a)=\pi(a)+q(a)\left(1-n_{H}\right) p(a)
$$

Note that this probability is always higher than $\pi(a)$.

Additional assumptions. We assume finally that $n_{H}<1$ and $q(e)>0$. If $n_{H}=1$, then it is not possible to have a high performance and a low result, thus making the provision of an excuse pointless. If $q(e)=0$, providing additional information is a certain proof of shirking. In this case, it would never be optimal to reward the agent who provides an excuse. Moreover, we will assume, when the agent has an unlimited liability (Sections 4 and $5)$, that $p(e) q(e)<1$, otherwise $\varphi(e)=1$, which implies that the policy of adjusting is always optimal with an agency cost equal to zero since it is in this case possible to provide incentives to the agent without placing any risk on him.

\section{Unlimited liability and complete contracting}

In this section, we make two assumptions. First, the principal can punish the agent with an arbitrarily large negative wage: the agent has a potentially unlimited liability. Second, the principal may differentiate the wages in the event additional information is provided by the agent: she can use a contract as complete as she wants. 
Given the binary nature of both $x$ and $y$, there are four possible wages. They depend on the result $y^{H}$ or $y^{L}$ and on the eventual provision of additional information by the agent proving that the performance is $x^{H}$, denoted $i$, where $i$ stands either for $\epsilon$ or $\eta$. The wages are denoted $w_{H}, w_{L}$ (if the agent does not provide evidence that the performance is $x^{H}$ ) and $w_{H}^{i}, w_{L}^{i}$ otherwise. Using the revelation principle, we restrict our attention to direct truthful mechanisms; that is, contracts for which $w_{H}^{i} \geq w_{H}$ and $w_{L}^{i} \geq w_{L}$.

The principal rewards the provision of information by the agent when either a low result with an excuse is more rewarded than a low result alone, $w_{L}^{i}>w_{L}$, or a high result with additional information is more rewarded than a high result alone, $w_{H}^{i}>w_{H}$. When such is the case, we will say that the policy of adjusting for uncontrollable factors is optimal.

The program to solve for the principal is (using $u_{j}$ instead of $u\left(w_{j}\right)$ for notational simplicity):

$$
\begin{aligned}
\min _{w_{H}^{i}, w_{L}^{i}, w_{H}, w_{L}} p(e) q(e) n_{H} w_{H}^{i} & +p(e) q(e)\left(1-n_{H}\right) w_{L}^{i} \\
& +\left(\pi(e)-p(e) q(e) n_{H}\right) w_{H}+(1-\varphi(e)) w_{L}
\end{aligned}
$$

under the constraints:

$$
\begin{gathered}
(p(e) q(e)-p(0) q(0))\left[n_{H}\left(u_{H}^{i}-u_{H}\right)+\left(1-n_{H}\right)\left(u_{L}^{i}-u_{L}\right)\right] \\
+(\pi(e)-\pi(0))\left(u_{H}-u_{L}\right) \geq C \\
p(e) q(e)\left[n_{H}\left(u_{H}^{i}-u_{H}\right)+\left(1-n_{H}\right)\left(u_{L}^{i}-u_{L}\right)\right] \\
+\pi(e) u_{H}+(1-\pi(e)) u_{L}-C \geq u_{0} \\
u_{H}^{i} \geq u_{H} \\
u_{L}^{i} \geq u_{L}
\end{gathered}
$$

Proposition 1. If $R^{\varepsilon}>R^{H}$, adjusting is always optimal, with $w_{H}^{i}=w_{L}^{i}>$ $w_{H}$.

If $R^{L}<R^{\varepsilon}<R^{H}$, adjusting is always optimal, with $w_{H}^{i}=w_{H}>w_{L}^{i}>w_{L}$. If $R^{\varepsilon} \leq R^{L}$, adjusting is never optimal.

When the opportunity cost of generating information is low, if $R^{\varepsilon}$ be- 
comes sufficiently large such that $R^{\varepsilon}>R^{H}$, then the agent has incentive to provide not only legitimate excuses because $w_{L}^{i}>w_{L}$ but also evidence that a high result is not a windfall since $w_{H}^{i}>w_{H}$. He receives the same reward when he provides additional information, independently of whether the result is high or low $\left(w_{H}^{i}=w_{L}^{i}\right)$, since, as already mentioned in Section 4 , the agent does not control the garbling between the performance and the result. When the agent is able to prove that $x=x^{H}$, the most informative state is attained and thus deserves the highest possible wage.

If $\left.R^{\varepsilon} \in\right] R^{L}, R^{H}$, it is optimal to reward the provision of an excuse, but with a lower wage than a high uncorrected result $\left(w_{H}>w_{L}^{i}\right)$. The principal does filter the effect of the performance measure, but the reward is lower. Furthermore, the agent has no incentive to provide additional information if the result is high $\left(w_{H}^{i}=w_{H}\right)$.

If the opportunity cost is high, the provision of information is the least informative state: $R^{\varepsilon}<R^{L}$, and thus it is optimal not to reward it.

\section{$5 \quad$ Unlimited liability and incomplete contracting}

The analysis developed in the previous section relied on the assumption that the principal can offer different rewards depending on whether the result has been corrected or not. In some circumstances, these differentiated rewards are not feasible. In this section, we assume therefore that the principal is restricted to offering the same reward in the case the uncorrected result is high or an excuse is provided proving that the performance is high. Using the notation of previous section: $w_{L}^{i}=w_{H}=w_{H}^{i}$. Note that there is no longer incentive to prove that a high result is not a windfall. Only excuses will be provided by the agent. This event is denoted $\epsilon$.

The principal must choose between an excuse contract in which legitimate excuses are rewarded and a no-excuse contract in which no adjustment is ever made. Let $w_{H}, w_{L}$ be the wages in the no-excuse contract. There are now only two possible wages if excuses are rewarded: $w_{L}^{\epsilon}$ if the uncorrected result is low and the agent does not provide an excuse; and $w_{H}^{\epsilon}$ when the result is high, or if the result is low and an excuse is provided. 
If the principal decides to use a no-excuse contract, her program is (again using $u_{j}$ instead of $\left.u\left(w_{j}\right)\right)$ :

$$
\min _{w_{H}, w_{L}} \pi(e) w_{H}+(1-\pi(e)) w_{L}
$$

under the constraints:

$$
\begin{gathered}
\pi(e) u_{H}+(1-\pi(e)) u_{L}-C \geq \pi(0) u_{H}+(1-\pi(0)) u_{L} \\
\pi(e) u_{H}+(1-\pi(e)) u_{L}-C \geq u_{0}
\end{gathered}
$$

The first constraint is the incentive compatibility constraint, the second one the individual rationality constraint.

If the principal commits to the excuse contract, her program is identical except that $\varphi($.$) is used in place of \pi($.$) so that the probability of obtaining$ $w_{H}^{\epsilon}$ is now $\varphi(a)$ and for the wages $w_{H}^{\epsilon}, w_{L}^{\epsilon}$ in place of $w_{H}, w_{L}$.

For notational simplicity, let $g$ stand for either $\pi$ or $\varphi$, and $w_{H}^{g}$ stand for $w_{H}$ or $w_{H}^{\varepsilon}$; and $w_{L}^{g}$ represent $w_{L}$ or $w_{L}^{\varepsilon}$, according to whether the principal uses an excuse or a no-excuse contract.

Lemma 1. The optimal wages depending on the contract $(g)$ selected by the principal are:

$$
\begin{aligned}
w_{L}^{g} & =u^{-1}\left(u_{0}-g(0) C /(g(e)-g(0))\right) \\
w_{H}^{g} & =u^{-1}\left(u_{0}+(1-g(0)) C /(g(e)-g(0))\right)
\end{aligned}
$$

The reward for the low result, $w_{L}^{g}$, depends, positively, on the likelihood ratio of an effort for the high result, $g(e) / g(0)$. The reward for the high result, $w_{H}^{g}$, can be rewritten as $u^{-1}\left(u_{0}+\frac{C}{1-(1-g(e)) /(1-g(0))}\right)$ and thus depends positively on the likelihood ratio for the low result, $(1-g(e)) /(1-g(0))$.

Thus, the comparison of the rewards from the no-excuse and the excuse contracts reverts to the comparison of their likelihood ratios. As the next lemma shows, this can be done by positioning the likelihood ratio $R^{\varepsilon}$ with respect to $R^{H}$ and $R^{L}$. 


\section{Lemma 2.}

$$
\begin{aligned}
& w_{L}^{\varepsilon} \leq w_{L} \quad \Leftrightarrow \quad \frac{\varphi(e)}{\varphi(0)} \leq \frac{\pi(e)}{\pi(0)} \quad \Leftrightarrow \quad R^{\varepsilon} \leq R^{H} \\
& w_{H}^{\varepsilon} \leq w_{H} \quad \Leftrightarrow \quad \frac{1-\varphi(e)}{1-\varphi(0)} \leq \frac{1-\pi(e)}{1-\pi(0)} \quad \Leftrightarrow \quad R^{\varepsilon} \geq R^{L}
\end{aligned}
$$

The first equivalence in Lemma 2 shows that the excuse contract is less informative than the no-excuse one for a high result, $\varphi(e) / \varphi(0) \leq \pi(e) / \pi(0)$, if and only if the provision of an excuse is less informative than a high uncorrected result, $R^{\varepsilon} \leq R^{H}$. For a low result, the most informative technology is the one with the higher likelihood ratio of shirking, or equivalently the lower likelihood ratio of making an effort. Thus, the second equivalence in Lemma 2 states that the excuse contract is more informative for a low result if and only if the provision of an excuse is more informative than a low uncorrected result, $R^{\varepsilon} \geq R^{L}$.

Since $R^{H} \geq R^{L}$, if $R^{\varepsilon} \geq R^{H}$, we also have $R^{\varepsilon} \geq R^{L}$. When this condition holds, Lemma 2 indicates that the spread of wages is lower with the excuse contract: $w_{L} \leq w_{L}^{\varepsilon} \leq w_{H}^{\varepsilon} \leq w_{H}$. At the other extreme, if $R^{\varepsilon} \leq R^{L}$, the reverse is true - the spread of wages is lower with the no-excuse contract: $w_{L}^{\varepsilon} \leq w_{L} \leq w_{H} \leq w_{H}^{\varepsilon}$.

As the agent is risk-averse, the higher the spread, the higher the incentive cost. Thus, in both cases it is possible to rank unambiguously the contracts. ${ }^{2}$ Proposition 2 is a direct extension of Proposition 1 for the extreme values of $R^{\epsilon}$.

Proposition 2. If $R^{\varepsilon} \geq R^{H}$, the excuse contract is always optimal. If $R^{\varepsilon} \leq R^{L}$, the no-excuse contract is always optimal.

Consider now the intermediate case. There are two opposite effects. First, because the provision of an excuse is less informative than a high un-

\footnotetext{
${ }^{2}$ This proposition is actually a particular case of a more general result shown by Kim (1995). When the likelihood ratio distribution of an information system $t$ is a mean preserving spread of the likelihood ratio distribution of another information system $h$, then the principal always prefers $t$, regardless of the preference of the agent (the utility function). When $R^{\varepsilon} \geq R^{H}$ and $R^{\varepsilon} \leq R^{L}$, it is possible to order both monitoring technologies, excuse and no-excuse, using the MPS criterion.
} 
corrected result, rewarding excuses increases the likelihood ratio of giving a windfall bonus to an agent who has shirked. This effect increases the incentive cost. Second, because the provision of an excuse is more informative than a low uncorrected result, rewarding excuses decreases the likelihood ratio of punishing an agent who made an effort. This effect decreases the incentive cost. The balance between the two effects depends on the specific utility function of each agent, as the next proposition shows.

Proposition 3. If $R^{L}<R^{\varepsilon}<R^{H}$, either contract may be optimal.

In particular, if the utility function is $u(x)=x^{\alpha} / \alpha$, with $\alpha \leq 1$, there is a limit $\alpha_{0}$ such that $\forall \alpha \leq \alpha_{0}$, the excuse contract is optimal.

Proposition 3 shows that the higher the risk aversion of the agent (that is, the lower $\alpha$ ), the more costly is the risk of punishing an agent who has made an effort, as compared to the cost of the potential windfall bonus. The excuse contract is in this case more likely to be optimal. ${ }^{3}$

An interesting remark can be made. Intuitively, if the agent is more likely to provide an excuse when he shirks $\left(R^{\varepsilon}<1\right)$, then there should be no point to rewarding the excuses. However, since $R^{L}<1$, the excuse contract might still be optimal in that case. The explanation is that the benefit of a contract that rewards excuses occurs only when the agent is unable to provide an excuse. The fact that the result is low and that the agent is unable to provide an excuse is very informative of shirking. The risk of punishing an agent who has made an effort and gets a low result is smaller. Thus, the excuse contract may be optimal even if providing an excuse is bad news in the sense that the agent is more likely to provide an excuse when he shirks.

\section{Limited liability}

In this section, we assume that the agent has a limited liability and that this constraint is binding at equilibrium. The principal would like to offer a neg-

\footnotetext{
${ }^{3}$ We were not able to show the converse of the second part of Proposition 3: $\forall \alpha>\alpha_{0}$, that the excuse contract is optimal. However, a great number of numerical simulations leads us to conjecture that the converse always holds.
} 
ative wage in some cases, but she cannot: all wages must be equal or above zero. This assumption fits well the incentive structure inside companies: the principal is limited in the possible punishment she can impose on the agent - it is unusual to ask the agent to give money to the firm, and most of the incentive must come from the bonus. We will look at two cases in sequence: complete contracting (counterpart of Section 4, but with limited liability), incomplete contracting (counterpart of Section 5).

\subsection{Complete contract}

Since $w_{H}^{i} \geq w_{H}$ and $w_{L}^{i} \geq w_{L}$, due to the restriction to direct truthful mechanisms, only two constraints need be added to the program of the principal stated in Section 4.

$$
\begin{aligned}
& w_{L} \geq 0 \\
& w_{H} \geq 0
\end{aligned}
$$

The latter constraint clearly cannot bind at equilibrium, otherwise we would have a fixed-wage contract.

Proposition 4. If $R^{\varepsilon}>R^{H}$, adjusting is always optimal, with $w_{H}^{i}=w_{L}^{i}>$ $w_{H}$.

If $1<R^{\varepsilon}<R^{H}$, the optimal policy, adjusting or not, depends on the risk aversion of the agent.

If $R^{\varepsilon} \leq 1$, adjusting is never optimal.

When $1<R^{\varepsilon}<R^{H}$, as demonstrated by the proof, we can say more about the choice of the principal. If the utility function of the agent is $u(x)=\left(x+x_{0}\right)^{\alpha} / \alpha$, with $0 \leq \alpha \leq 1$ and $x_{0}>0$, there exists a cutoff $\alpha_{1}$ such that rewarding the providing of information is optimal if and only if $\alpha<\alpha_{1}$. That is, the policy of adjusting is optimal if and only if the agent is sufficiently risk averse. The assumption $x_{0}>0$ is crucial. If $x_{0}=0$, that is, if we were using the utility function of Proposition 3, then the agent is infinitely risk averse close to minimum wage zero. In that case, rewarding excuses, even with a small wage, is always optimal. 
The introduction of limited liability reduces the range over which adjusting is optimal. Comparing with the complete contracting case with unlimited liability (Proposition 1 of Section 4), the range over which it is always optimal not to reward the excuse is enlarged. Firstly, we have $R^{L}<1$. Thus, inside the bracket $] R^{L}, 1[$, rewarding excuses is never optimal when the limited liability binds, whereas the reverse is true: rewarding excuses is always optimal when the limited liability does not bind.

Secondly, above 1 and below $R^{H}$, not rewarding excuse might still be optimal in the limited-liability case; whereas it is never the case with unlimited liability of the agent.

Thus, when the limited liability is binding, there is a shift in favor of not adjusting. Indeed, with limited liability, the agent earns rent, which generates an additional agency cost on top of the risk premium necessary for the agent to accept to bear risks. For a monitoring technology $g$, this rent is equal to $g(0) C /(g(e)-g(0))-u_{0}$ and thus depends (negatively) on the informativeness of a high outcome. When $R^{\varepsilon} \leq R^{H}$, the no-excuse contract generates a lower rent than the excuse contract and thus is more often optimal than with unlimited liability.

\subsection{Incomplete contracting}

Again, we need to add the limited liability constraint $w_{L} \geq 0$ to the programs of Section 5. Recall that $g$ stands for either $\pi$ or $\varphi$, and $w_{H}^{g}\left(w_{L}^{g}\right)$ stands for $w_{H}$ or $w_{H}^{\varepsilon}$ (respectively $w_{L}$ or $w_{L}^{\varepsilon}$ ). The limited liability constraint is binding if and only if $u_{0} \leq g(0) C /(g(e)-g(0))$. We suppose that this condition holds throughout this section for at least one of the monitoring technologies. We now have the equivalent to Lemma 1.

Lemma 3. If the limited liability binds, the optimal wages depending on the contract $(g)$ selected by the principal are:

$$
\begin{aligned}
& w_{H}^{g}=u^{-1}(C /(g(e)-g(0)) \\
& w_{L}^{g}=0
\end{aligned}
$$


Here we have a counterpart to Propositions 2 and 3.

Proposition 5. If $R^{\varepsilon} \geq R^{H}$, the excuse contract is always optimal. If $R^{\varepsilon} \leq 1$, the no-excuse contract is always optimal.

Proposition 6. If $1<R^{\varepsilon}<R^{H}$ and the utility function is $u(x)=x^{\alpha} / \alpha$, with $0 \leq \alpha \leq 1$, there is a limit $\left.\alpha_{2} \in\right] 0,1[$ such that the excuse contract is optimal if and only if $\alpha \leq \alpha_{2}$.

Note that, as in the complete contracting case, $R^{L}$ is now replaced by $1 .^{4}$

When the limited liability constraint binds, results are very similar whether or not the principal is restricted to using only a bonus function: for intermediate values of $R^{\epsilon}$, the optimal contract depends on the risk aversion of the agent. This configuration is in sharp contrast with the case of unlimited liability (again, see Figure 2).

Moreover, in this case, a very straightforward explanation of why it is never optimal to reward excuses when $R^{\epsilon}<1$ can be provided, assuming that the limited liability binds for both contracts. As $\varphi(e) \geq \pi(e)$, the bonus is always paid more often with the excuse contract than with the no-excuse contract. If $R^{\epsilon}<1$, then the bonus of the excuse contract, given Lemma 3 , is higher. A higher bonus and paid more often: the excuse contract is clearly not optimal when $R^{\epsilon}<1$.

\subsection{Risk neutrality}

The limited liability constraint with a risk-neutral agent is often used as a substitute for the risk aversion of the agent as the source of the agency conflict with the principal. In this case, as the next proposition shows, only the likelihood ratio for the high outcome plays a role.

\footnotetext{
${ }^{4}$ The reason why 1 now replaces $R^{L}$ is explained in Larmande (2013). When the limited liability binds, the MPS criterion of Kim is no longer necessary to order two monitoring technologies, regardless of the utility function of the agent. A less stringent condition exists that involves the comparison of likelihood ratios for the high outcome and the comparison of marginal productivities of effort. The latter condition takes here the form of $R^{\epsilon}$ below 1 .
} 
Proposition 7. When the agent is risk-neutral and the wages are constrained through a limited liability condition, then the principal prefers to adjust if and only if $R^{\varepsilon} \geq R^{H}$.

Arya and Glover (2008) analyze an appeal model in which the agent is risk-neutral and protected by a limited liability. The agent has two tasks and can make an effort or not in each of them. The outcome is high only if the results of the two tasks are high. As in our model, the effort gives rise to a performance that is only observable by the agent. Arya and Glover consider two monitoring technologies. In the case where the outcome is not high, either the agent has the choice to cherry-pick which of the two tasks is appealed (i.e. the agent can decide to provide a verifiable excuse for the task selected), or the two tasks are jointly appealed if the agent decides to appeal. A proposition similar to our Proposition 7 is proved. We conjecture that Arya and Glover's result does not extend directly to a risk-averse agent.

\subsection{Preference of the agent}

Consider now the preference of the agent regarding whether or not the principal should encourage the communication of additional information. It depends on the expected rent he may earn. With unlimited liability, this rent is zero, and thus the agent is indifferent. When the limited liability constraint binds this rent, as already mentioned, depends (negatively) on the informativeness of a high outcome. Thus, given Lemma 2, the agent prefers the excuse contract if and only if $R^{\epsilon} \leq R^{H}$. If the opportunity cost of generating an excuse is low, the agent prefers a result-based contract without any adjustments. On the contrary, when this cost is intermediate or high, the agent would prefer a contract that takes excuses into account.

In general, the preferences of the agent and of the principal are opposed: the rent is a direct transfer from the principal to the agent. There is one case in which their preferences might be aligned: within the interval $] 1, R^{H}[$; that is, when the opportunity cost of generating excuses assumes intermediate values. In this case, the agent prefers excuses to be taken into account because it generates a higher rent. The principal might also prefer the 
excuse contract, but for another reason: such higher rent is an influence cost that the principal accepts to pay because the risk borne by the agent is lower, and thus a lower risk premium is needed.

\section{Concluding remarks}

This research contributes to the understanding of the potential benefits and pitfalls of introducing a culture of excuses, giving due attention to practical constraints encountered in the design of the contract. Consider a situation in which the agent can convince the principal that his or her performance has been good in spite of an unfavorable environment. Also consider that equity requires that the allocation of a bonus be equal to the one the agent would have obtained for a high uncorrected result. Under these circumstances, we prove that the allowance of fishing for excuses, with the risk of rewarding the time spent on excuse building rather than on productive effort, might still be beneficial to the principal. This will be true only if the opportunity cost of making excuses, i.e. spending time on unproductive tasks to be in a good position to provide verifiable evidence, is not too large. Risk aversion of the agent extends the range over which the excuse contract is optimal. These results have been proved to hold in a context of unlimited or limited liability. They also hold in a context in which the principal may differentiate the wages in the event the agent provides additional information. The exact boundaries that characterize the range under which each form of contract is optimal has been precisely delineated. We also provide predictions for the preference of the agent regarding this issue. Altogether the model provides a number of predictions which will be worth testing in an empirical setting.

Our formal analysis has several limits that deserve further research. First, we have assumed no asymmetry of information regarding the probability of gathering verifiable evidence. Relaxing this assumption for the excuse contract might lead to separating equilibria where low performing agents might be asked not to work. We conjecture that our result may still be valid when the asymmetry of information is small. Second, we assume no moral hazard on the principal's side. Relaxing this assumption and analyzing the 
fishing for excuses in a relational contract, as in Macleod (2003), might yield interesting results. Third, the model is a simple binomial model. Does our result hold in a multinomial or a continuous model? Arya and Glover (2008) find similar results in a trinomial model, but more work needs to be done to assess the generality of the result.

Finally, the model assumes that the performance evaluation is performed directly by the principal or by an independent internal or external auditor. In practice, this evaluation may also be delegated to a supervisor, for instance the direct line manager of the agent. This evaluation can itself be plagued by moral hazard. The policy of adjusting, based on a subjective assessment by a supervisor, would be more prone to moral hazard and collusion than the no-excuse contract, based on information provided through the company's control system. This effect can reduce the potential benefits of rewarding excuses.

\section{References}

Arya, A. \& Glover, J. (2008). Performance measurement manipulation: cherry-picking what to correct. Review of Accounting Studies, 13, 119139.

Demski, J. (1998). Performance measure manipulation. Contemporary Accounting Research, 15, 261-285.

Demski, J., Frimor, H. \& Sappington, D. (2004). Efficient manipulation in a repeated setting. Journal of Accounting Research, 42, 31-50.

Dutta, S. \& Gigler, F. (2002). The effect of earnings forecasts on earnings management. Journal of Accounting Research, 40, 631-655.

Feltham, G. \& Xie, J. (1994). Performance measure congruity and diversity in multi-task principal/agent relations. The Accounting Review, 429-453.

Fudenberg, D. \& Tirole, J. (1990). Moral hazard and renegotiation in agency contracts. Econometrica, 58, 1279-1320. 
Kim, S. K. (1995). Efficiency of an information system in an agency model. Econometrica, 63, 89-102.

Larmande, F. (2013). Limited liability, the first-order approach, and the ranking of information systems in agencies. Economics Letters, 118, 314317.

Larmande, F. \& Ponssard, J.P. (2008). Implementation of an EVA compensation scheme, 69-98, in Reward management - facts and trends in Europe. Lengerich: Pabst.

MacLeod, B. (2003). Optimal contracting with subjective evaluation. The American Economic Review, 93, 216-240.

Merchant, K. \& Van der Stede, W. (2003). Management control systems. Harlow: Prentice Hall.

Milgrom, P. \& Roberts, J. (1988). An economic approach to influence activities in organizations. American Journal of Sociology, 94, 154-179. 


\section{Appendix}

\section{Proof of Lemma 1}

Both the incentive compatibility constraint and the individual rationality constraint are binding, leading to the derivation of the optimal wages.

\section{Proof of Lemma 2}

From Lemma $1 w_{L}^{\epsilon} \leq w_{L}$ if and only if

$$
\frac{\varphi(e)}{\varphi(0)} \leq \frac{\pi(e)}{\pi(0)}
$$

By definition $\frac{\varphi(e)}{\varphi(0)}=\frac{\pi(e)+q(e) p(e)\left(1-n_{H}\right)}{\pi(0)+q(0) p(0)\left(1-n_{H}\right)}$, so that

$$
\frac{\varphi(e)}{\varphi(0)} \leq \frac{\pi(e)}{\pi(0)} \Leftrightarrow \frac{q(e) p(e)\left(1-n_{H}\right)}{q(0) p(0)\left(1-n_{H}\right)} \leq \frac{\pi(e)}{\pi(0)} \Leftrightarrow R^{\epsilon} \leq R^{H}
$$

From Lemma $1 w_{H}^{\epsilon} \leq w_{H}$ if and only if $\frac{1-\varphi(e)}{1-\varphi(0)} \leq \frac{1-\pi(e)}{1-\pi(0)}$. That is,

$$
\begin{aligned}
\frac{1-\pi(e)-q(e) p(e)\left(1-n_{H}\right)}{1-\pi(0)-q(0) p(0)\left(1-n_{H}\right)} & \leq \frac{1-\pi(e)}{1-\pi(0)} \\
\Leftrightarrow(1-\pi(0))\left(-q(e) p(e)\left(1-n_{H}\right)\right) & \leq(1-\pi(e))\left(-q(0) p(0)\left(1-n_{H}\right)\right) \\
\Leftrightarrow \frac{1-\pi(e)}{1-\pi(0)} & \leq R^{\epsilon} \\
\Leftrightarrow R^{L} & \leq R^{\epsilon}
\end{aligned}
$$

\section{Proof of Proposition 1}

Let $\lambda_{1}, \lambda_{2}, \mu_{1}$ and $\mu_{2}$ denote the Lagrange multipliers for the four constraints of the program of the principal. The objective function of the principal can be rewritten as

$$
p(e) q(e)\left[n_{H}\left(w_{H}^{i}-w_{H}\right)+\left(1-n_{H}\right)\left(w_{L}^{i}-w_{L}\right)\right]+\pi(e) w_{H}+(1-\pi(e)) w_{L}
$$

Because $n_{H}<1$ and $n_{H}>0$ we can multiply (8) by $n_{H}$ and (9) by 
$\left(1-n_{H}\right)$.The first-order Lagrangian conditions are:

$$
\begin{gathered}
p(e) q(e) f^{\prime}\left(u_{H}^{i}\right)=(p(e) q(e)-p(0) q(0)) \lambda_{1}+p(e) q(e) \lambda_{2}+\mu_{1} \\
\left(\pi(e) / n_{H}-p(e) q(e)\right) f^{\prime}\left(u_{H}\right)=-(p(e) q(e)-p(0) q(0)) \lambda_{1}-p(e) q(e) \lambda_{2} \\
-\mu_{1}+(\pi(e)-\pi(0)) / n_{H} \lambda_{1}+\pi(e) / n_{H} \lambda_{2} \\
p(e) q(e) f^{\prime}\left(u_{L}^{i}\right)=(p(e) q(e)-p(0) q(0)) \lambda_{1}+p(e) q(e) \lambda_{2}+\mu_{2} \\
(1-\varphi(e)) /\left(1-n_{H}\right) f^{\prime}\left(u_{L}\right)=-(p(e) q(e)-p(0) q(0)) \lambda_{1}-p(e) q(e) \lambda_{2}-\mu_{2} \\
-(\pi(e)-\pi(0)) /\left(1-n_{H}\right) \lambda_{1}+(1-\pi(e)) /\left(1-n_{H}\right) \lambda_{2}
\end{gathered}
$$

Adding (10) and (11) we obtain

$$
p(e) q(e) n_{H}\left(f^{\prime}\left(u_{H}^{i}\right)-f^{\prime}\left(u_{H}\right)\right)+\pi(e) f^{\prime}\left(u_{H}\right)=(\pi(e)-\pi(0)) \lambda_{1}+\pi(e) \lambda_{2}
$$

Because $f^{\prime}$ increases (as $f$ is convex) and because $u_{H}^{i} \geq u_{H}$, we obtain

$$
\pi(e) f^{\prime}\left(u_{H}\right) \leq(\pi(e)-\pi(0)) \lambda_{1}+\pi(e) \lambda_{2}
$$

Combining this latter inequality with (11) and noting that $\pi(e) / n_{H}-p(e) q(e) \geq$ 0 , we obtain

$$
\mu_{1} \geq(p(0) q(0)-p(e) q(e) \pi(0) / \pi(e)) \lambda_{1}
$$

If $R^{\epsilon}<R^{H}$, then by (15), either $\mu_{1}>0$ or $\lambda_{1}=\mu_{1}=0$. In the latter case (10) and (11) imply that $f^{\prime}\left(u_{H}^{i}\right)=\lambda_{2}=f^{\prime}\left(u_{H}\right)$. Thus, if $R^{\epsilon}<R^{H}$, then $u_{H}^{i}=u_{H}$.

Conversely, assume that $u_{H}^{i}=u_{H}$. Then (14) and (15) hold with equality. If $R^{\epsilon}>R^{H}$, then $p(0) q(0)-p(e) q(e) \pi(0) / \pi(e)<0$. The only possibility is that $\mu_{1}=\lambda_{1}=0$. Then (10) and (12) (and the fact that $w_{H}^{i} \geq w_{L}^{i}$ ) imply that $f^{\prime}\left(u_{H}^{i}\right)=f^{\prime}\left(u_{L}^{i}\right)=\lambda_{2}$ and $\mu_{2}=0$. Because $\mu_{1}=\lambda_{1}=\mu_{2}=0$, (13) becomes $f^{\prime}\left(u_{L}\right)=\lambda_{2}$. Thus, all wages are equal in this case, which is impossible. Therefore if $R^{\epsilon}>R^{H}$, we have $u_{H}^{i}>u_{H}$.

The same reasoning can be used for the low outcome. Adding (12) and 
(13) leads to:

$$
f^{\prime}\left(u_{L}\right) \leq-\frac{\pi(e)-\pi(0)}{1-\pi(e)} \lambda_{1}+\lambda_{2}
$$

Substituting into (13) we obtain:

$$
\mu_{2} \geq(p(0) q(0)-p(e) q(e)(1-\pi(0)) /(1-\pi(e))) \lambda_{1}
$$

Following the same reasoning as above, if $R^{\epsilon}>R^{L}$, then $u_{L}^{i}>u_{L}$. And if $R^{\epsilon}<R^{L}$, then $u_{L}^{i}=u_{L}$, which concludes the proof.

\section{Proof of Proposition 2}

As a first step, we are going to show that, given two monitoring technologies $t$ and $h$, if the following two inequalities hold,

$$
\begin{aligned}
\frac{1-t(e)}{1-t(0)} & \leq \frac{1-h(e)}{1-h(0)} \\
\frac{h(e)}{h(0)} & \leq \frac{t(e)}{t(0)}
\end{aligned}
$$

it will be proved that the principal always prefers $t$ to $h$, whatever the utility function of the agent.

Denote $u_{H}^{h}=u_{0}+(1-h(0)) C /(h(e)-h(0))$ and $u_{L}^{h}=u_{0}-h(0) C /(h(e)-$ $h(0))$. By construction $w_{L}^{h}=u^{-1}\left(u_{L}^{h}\right)$ and $w_{H}^{h}=u^{-1}\left(u_{H}^{h}\right)$. Symmetrically, denote $u_{H}^{t}=u\left(w_{H}^{t}\right)$ and $u_{L}^{t}=u\left(w_{L}^{t}\right)$.

Lemma 2 shows that $(17) \Leftrightarrow u_{H}^{t} \leq u_{H}^{h}$ and $(18) \Leftrightarrow u_{L}^{h} \leq u_{L}^{t}$.

As the individual rationality constraint is binding, $h(e) u_{H}^{h}+(1-h(e)) u_{L}^{h}=$ $t(e) u_{H}^{t}+(1-t(e)) u_{L}^{t}=u_{0}+C$. Thus, $h$ increases the spread of utilities necessary to provide the incentive to the agent $\left(u_{L}^{h} \leq u_{L}^{t} \leq u_{H}^{t} \leq u_{H}^{h}\right)$ while keeping the same mean (expected utility). As $u^{-1}$ is convex:

$$
h(e) u^{-1}\left(u_{H}^{h}\right)+(1-h(e)) u^{-1}\left(u_{L}^{h}\right) \geq t(e) u^{-1}\left(u_{H}^{t}\right)+(1-t(e)) u^{-1}\left(u_{L}^{t}\right)
$$

that is, the incentive cost associated with $h$ is higher than the cost associated with $t$. Thus the principal always prefers $t$ to $h$.

As a second step, on the one hand, if $R^{\epsilon} \geq R^{H}$, Lemma 2 shows that 
$\frac{1-\varphi(e)}{1-\varphi(0)} \leq \frac{1-\pi(e)}{1-\pi(0)}$ and $\frac{\pi(e)}{\pi(0)} \leq \frac{\varphi(e)}{\varphi(0)}$. The principal always prefers $\varphi$, that is, the excuse contract.

On the other hand, if $R^{\epsilon} \leq R^{L}$, then Lemma 2 shows that the inequalities are in reverse order. The no-excuse contract is always optimal.

\section{Proof of Proposition 3}

Denote $u_{L}^{\epsilon}=u\left(w_{L}^{\epsilon}\right)=u_{0}-\varphi(0) C /(\varphi(e)-\varphi(0)), u_{H}^{\epsilon}=u\left(w_{H}^{\epsilon}\right)=u_{0}+$ $(1-\varphi(0)) C /(\varphi(e)-\varphi(0)), u_{L}=u\left(w_{L}\right)=u_{0}-\pi(0) C /(\pi(e)-\pi(0))$ and, $u_{H}=u\left(w_{H}\right)=u_{0}+(1-\pi(0)) C /(\pi(e)-\pi(0))$. Let $I C^{\epsilon}$ and $I C$ denote the respective expected wages paid by the principal (the minimum of the objective function of the principal).

Lemma 2 can be directly extended to show that $R^{\epsilon}<R^{H} \Leftrightarrow \varphi(e) / \varphi(0)<$ $\pi(e) / \pi(0) \Leftrightarrow u_{L}^{\epsilon}<u_{L}$, and that $R^{\epsilon}>R^{L} \Leftrightarrow u_{H}^{\epsilon}<u_{H}$.

Because the participation constraint is binding,

$$
\pi(e) u_{H}+(1-\pi(e)) u_{L}=u_{0}+C=\varphi(e) u_{H}^{\epsilon}+(1-\varphi(e)) u_{L}^{\epsilon}
$$

Example where the no-excuse contract is optimal. Consider the following function $u^{-1}: u^{-1}(x)=u_{L}$ for $x \leq u_{L}$, and $u^{-1}(x)=x$ for $x>u_{L}$. Then, $I C=\pi(e) u_{H}+(1-\pi(e)) u_{L}=u_{0}+C$ and $I C^{\epsilon}=\varphi(e) u_{H}^{\epsilon}+(1-\varphi(e)) u_{L}=$ $u_{0}+C+(1-\varphi(e))\left(u_{L}-u_{L}^{\epsilon}\right)$. Since $\varphi(e)<1$ and $u_{L}-u_{L}^{\epsilon}$, we obtain therefore $I C^{\epsilon}>I C$.

Example where the excuse contract is optimal. Consider the specific utility function $u(x)=x^{\alpha} / \alpha$. The excuse contract is optimal if and only if the expected wage is lower, that is,

$$
\pi(e)\left(u_{H}\right)^{1 / \alpha}+(1-\pi(e))\left(u_{L}\right)^{1 / \alpha}-\varphi(e)\left(u_{H}^{\epsilon}\right)^{1 / \alpha}-(1-\varphi(e))\left(u_{L}^{\epsilon}\right)^{1 / \alpha} \geq 0
$$

Let $\Delta(\alpha)$ denote the LHS of the above inequality. As $u_{L}^{\epsilon}<u_{L}<u_{H}^{\epsilon}<u_{H}$ (because of Lemma 2), $\Delta(\alpha) /\left(u_{H}\right)^{1 / \alpha}$ goes to $\pi(e)$ when $\alpha$ goes to 0 . As $\pi(e)>0$, there is an $\alpha_{0}$ such that $\forall \alpha \leq \alpha_{0}, \Delta(\alpha) /\left(u_{H}\right)^{1 / \alpha}>0$. Thus, $\forall \alpha \leq \alpha_{0}$ the excuse contract is optimal. 


\section{Proof of Proposition 4}

First if $R^{\epsilon}>R^{H}$ (resp. $R^{\epsilon}<R^{L}$ ), a proof similar to the proof of Proposition 1 show that adjusting is always optimal (resp. adjusting is never optimal). Assume from now on that $\left.R^{\epsilon} \in\right] R^{L}, R^{H}$.

The limited liability is binding, thus $w_{L}=0$ and the individual rationality constraint is slack. Moreover, for the same informativeness reason than in the unlimited liability case, as $R^{\epsilon}<R^{H}, u_{L}^{i} \leq u_{H}$ and $u_{H}^{i}=u_{H}$. As a result $u_{H}>u(0)$ otherwise the incentive constrained is not satisfied.

We can rewrite the program as follows:

$$
\min _{u_{L}^{i}, u_{H}} p(e) q(e)\left(1-n_{H}\right) f\left(u_{L}^{i}\right)+\pi(e) f\left(u_{H}\right)
$$

under the constraints

$$
\begin{gathered}
(p(e) q(e)-p(0) q(0))\left(1-n_{H}\right)\left(u_{L}^{i}-u(0)\right)+(\pi(e)-\pi(0))\left(u_{H}-u(0)\right) \geq C \\
u_{L}^{i} \geq 0
\end{gathered}
$$

Denote respectively $\lambda$ and $\mu$ the Lagrange multipliers associated with the two constraints. The first order Lagrangian conditions are:

$$
\begin{aligned}
\pi(e) f^{\prime}\left(u_{H}\right) & =\lambda(\pi(e)-\pi(0)) \\
p(e) q(e)\left(1-n_{H}\right) f^{\prime}\left(u_{L}^{i}\right) & =\lambda(p(e) q(e)-p(0) q(0))\left(1-n_{H}\right)+\mu
\end{aligned}
$$

If $R^{\epsilon}<1$ then $p(e) q(e) \leq p(0) q(0)$. Moreover since $f$ is strictly increasing, $\lambda$ is always strictly positive. The second Lagrangian constraint gives $\mu>0$ : excuses are never taken into account. By continuity it is also the case if $R=1$.

Assume now that $R^{\epsilon}>1$ and that the utility function has a power shape $u(x)=\left(x+x_{0}\right)^{\alpha} / \alpha$ with $x_{0}>0$. By combining the two Lagrangian conditions we obtain

$$
\mu=(p(e) q(e)-p(0) q(0))\left(1-n_{H}\right) \alpha^{1 / \alpha-1}\left(\frac{R^{\epsilon}}{R^{\epsilon}-1}\left(u_{L}^{i}\right)^{1 / \alpha-1}-\frac{R^{H}}{R^{H}-1}\left(u_{H}\right)^{1 / \alpha-1}\right)
$$


Denote

$$
\begin{aligned}
\mu_{0}=(p(e) q(e)-p(0) q(0))\left(1-n_{H}\right) \alpha^{1 / \alpha-1} \\
\\
\left(\frac{R^{\epsilon}}{R^{\epsilon}-1} u(0)^{1 / \alpha-1}-\frac{R^{H}}{R^{H}-1}(u(0)+C /(\pi(e)-\pi(0)))^{1 / \alpha-1}\right)
\end{aligned}
$$

As $u_{H} \leq u(0)+C /(\pi(e)-\pi(0))$ and $u_{L}^{i} \leq u(0), \mu \geq \mu_{0}$. Moreover $\mu_{0}$ can be rewritten as $(p(e) q(e)-p(0) q(0))\left(1-n_{H}\right)(\alpha u(0))^{1 / \alpha-1} \Phi(\alpha)$ in which $\Phi(\alpha)=\frac{R^{\epsilon}}{R^{\epsilon}-1}-\frac{R^{H}}{R^{H}-1}\left(\frac{u(0)+C /(\pi(e)-\pi(0))}{u(0)}\right)^{1 / \alpha-1}$.

Since $R^{\epsilon}<R^{H}$, we have $\Phi(1)>0$. On the other hand, when $\alpha$ goes to zero, $\Phi(\alpha)$ goes to $-\infty$. By the theorem of intermediate value, as $\Phi(\alpha)$ is continuous, there exists an $\left.\alpha_{1} \in\right] 0,1\left[\right.$ such that $\phi\left(\alpha_{1}\right)=0$. As $\Phi$ is strictly increasing with respect to $\alpha$ we obtain that $\Phi(\alpha)>0$ if $\alpha>\alpha_{1}$ and $\Phi(\alpha)<0$ if $\alpha<\alpha_{1}$.

If $\alpha>\alpha_{1}$, then $\mu \geq \mu_{0}>0$. Thus $u_{L}^{i}=u_{0}$ : excuses are never taken into account. By continuity, it is also the case if $\alpha=\alpha_{1}$.

If $\alpha<\alpha_{1}$, excuses are always taken into account. Assume the contrary. Then $u_{L}^{i}=u_{0}$ and the incentive constraint implies that $u_{H}=$ $u(0)+C /(\pi(e)-\pi(0))$. Thus $\mu=\mu_{0}<0$ which is impossible. Thus excuses are always taken into account.

We have shown that excuses are taken into account if and only if $\alpha<\alpha_{1}$.

Notice that, if $x_{0}=0$, then $\mu_{0}<0$, which means that excuses are always taken into account (otherwise, $u_{L}^{i}=u_{0}, u_{H}=u(0)+C /(\pi(e)-\pi(0)$ ), thus $\mu=\mu_{0}<0$ which is impossible).

\section{Proof of Proposition 5}

For a given monitoring technology $g$, let $I C_{l l}^{g}$ denote the incentive cost when the wages are 0 and $f(C /(g(e)-g(0)))$ (that is, the optimal wages when the limited liability binds) and $I C_{\text {unll }}^{g}$ when the wages are $f\left(u_{0}-g(0) C /(g(e)-g(0))\right)$ and $f\left(u_{0}+(1-g(0)) C /(g(e)-g(0))\right)$ (the optimal wages when the limited liability is omitted). Since the program in which the limited liability binds is more constrained than the program where this constraint is omitted, we have $I C_{l l}^{g} \geq I C_{u n l l}^{g}$. Denote $I C_{x}$ and $I C_{x}^{\epsilon}$, for $x \in\{l l$, unll $\}$ the incentive 
costs for the no-excuse contract and the excuse contract respectively.

Assume first that $R^{\epsilon}<1$. If both limited liability constraints bind then the no-excuse contract is always optimal because the bonus is lower (as $R^{\epsilon}<1$ and given Lemma 3) and paid less often (as $\pi(e) \leq \varphi(e)$ ). Thus, $I C_{l l} \leq I C_{l l}^{\epsilon}$. Moreover, as $R^{\epsilon}<R^{H}$, Lemma 2 shows that $\varphi(e) / \varphi(0) \leq$ $\pi(e) / \pi(0)$. Given the condition found in Lemma 3 , if only one of the limited liability constraints binds, that constraint is necessarily the one of the excuse contract. In that case, by combining $I C_{\text {unll }} \leq I C_{l l}$ and $I C_{l l} \leq I C_{l l}^{\epsilon}$, we obtain $I C_{\text {unll }} \leq I C_{l l}^{\epsilon}$. Thus, the no-excuse contract is also optimal when the limited liability binds for only one monitoring technology.

Assume now that $R^{\epsilon}>R^{H}$. Then $R^{\epsilon}>1$, and $\varphi(e)-\varphi(0) \geq \pi(e)-\pi(0)$.

Since $u^{-1}$ is a convex function, $\forall 0<x \leq y,\left(u^{-1}(y)-u^{-1}(0)\right) / y \geq$ $\left(u^{-1}(x)-u^{-1}(0)\right) / x$. Because $0<C /(\varphi(e)-\varphi(0)) \leq C /(\pi(e)-\pi(0))$, we obtain:

$$
\frac{u^{-1}(C /(\pi(e)-\pi(0)))-u^{-1}(0)}{u^{-1}(C /(\varphi(e)-\varphi(0)))-u^{-1}(0)} \geq \frac{\varphi(e)-\varphi(0)}{\pi(e)-\pi(0)}
$$

Moreover, because $R^{\epsilon}>R^{H}$, then $\pi(e) / \pi(0)<\varphi(e) / \varphi(0)$. Then:

$$
\frac{\varphi(e)-\varphi(0)}{\pi(e)-\pi(0)} \geq \frac{\varphi(e)}{\pi(e)}
$$

We obtain:

$$
\begin{aligned}
\pi(e) u^{-1}(C /(\pi(e) & -\pi(0)))+(1-\pi(e)) u^{-1}(0) \\
& \geq \varphi(e) u^{-1}(C /(\varphi(e)-\varphi(0)))(1-\varphi(e)) u^{-1}(0)
\end{aligned}
$$

Because $\pi(e) / \pi(0)<\varphi(e) / \varphi(0)$ and given the condition of Lemma 3, if the limited liability constraint binds for the excuse contract, then this constraint also binds for the no-excuse contract. Thus either both limited liability constraints bind, or only the limited liability constraint of the noexcuse contract binds.

Assume first that both limited liability constraints bind. Then (19) shows that the incentive cost is lower with the excuse contract.

Now assume that the limited liability constraint binds only for the no- 
excuse contract. As explained above, $I C_{l l}^{\epsilon} \geq I C_{\text {unll }}^{\epsilon}$ always holds. Moreover, (19) shows that $I C_{l l} \geq I C_{l l}^{\epsilon}$. Thus, we finally obtain $I C_{l l} \geq I C_{u n l l}^{\epsilon}$, and the excuse contract is always optimal.

\section{Proof of Proposition 6}

Since $R^{\epsilon}<R^{H}$, if only one limited liability constraint bind, it is the one of the excuse contract, as shown above. Assume first that the limited liability constraint also binds for the no-excuse contract. The no-excuse contract is optimal if and only if $\varphi(e) u^{-1}\left(\frac{C}{\varphi(e)-\varphi(0)}\right) \geq \pi(e) u^{-1}\left(\frac{C}{\pi(e)-\pi(0)}\right)$. As $u^{-1}(y)=(\alpha y)^{1 / \alpha}$, this condition is equivalent to

$$
\frac{\varphi(e)}{\pi(e)}-\left(\frac{\varphi(e)-\varphi(0)}{\pi(e)-\pi(0)}\right)^{1 / \alpha} \geq 0
$$

Let $\Delta(\alpha)$ denote the LHS of the above inequality. The no-excuse contract is optimal if and only if $\Delta(\alpha) \geq 0$. $\frac{\partial \Delta}{\partial \alpha}=\frac{1}{\alpha^{2}} \ln \left(\frac{\varphi(e)-\varphi(0)}{\pi(e)-\pi(0)}\right)\left(\frac{\varphi(e)-\varphi(0)}{\pi(e)-\pi(0)}\right)^{1 / \alpha}$.

As $R^{\epsilon}>1, \varphi(e)-\varphi(0)>\pi(e)-\pi(0)$. Thus $\frac{\partial \Delta}{\partial \alpha}>0: \Delta(\alpha)$ increases with respect to $\alpha$. Moreover, because $R^{\epsilon}<R^{H}$, then $\varphi(e) / \varphi(0)<\pi(e) / \pi(0)$ and thus $\Delta(1)>0$. And as $\varphi(e)-\varphi(0)>\pi(e)-\pi(0), \Delta(\alpha)$ goes to $-\infty$ when $\alpha$ goes to 0 . Given the intermediate value theorem, there exists a cutoff $\left.\alpha_{2} \in\right] 0,1\left[\right.$ such that $\Delta\left(\alpha_{2}\right)=0$. The monotonicity of $\Delta(\alpha)$ gives that the no-excuse contract is optimal if and only if $\alpha$ is above $\alpha_{2}$.

Assume now that the limited liability does bind only for the excuse contract. In that case, the no-excuse contract is optimal if and only if

$$
\begin{array}{r}
\varphi(e)\left(\alpha u_{H}^{\epsilon}\right)^{1 / \alpha}-\pi(e)\left(\alpha u_{H}\right)^{1 / \alpha}-(1-\pi(e))\left(\alpha u_{L}\right)^{1 / \alpha} \geq 0 \\
\Leftrightarrow \varphi(e)\left(u_{H}^{\epsilon} / u_{L}\right)^{1 / \alpha}-\pi(e)\left(u_{H} / u_{L}\right)^{1 / \alpha}-(1-\pi(e)) \geq 0
\end{array}
$$

Denote $\Gamma(\alpha)$ the LHS of the above inequality. As $R^{\epsilon}>1 \geq(1-\pi(e)) /(1-$ $\pi(0)$ ), following Lemma 2, we have $u_{H}^{\epsilon}<u_{H}$. Moreover, $u_{L}<u_{H}$. Thus $\Gamma(\alpha)\left(u_{L} / u_{H}\right)^{1 / \alpha}=\varphi(e)\left(u_{H}^{\epsilon} / u_{H}\right)^{1 / \alpha}-\pi(e)-(1-\pi(e))\left(u_{L} / u_{H}\right)^{1 / \alpha}$ which goes to $-\pi(e)<0$ when $\alpha$ goes to zero. As all wages are positive, $\Gamma(\alpha)$ is strictly negative for $\alpha$ close enough to zero. 
If $\alpha=1$ the agent is risk neutral and only the likelihood ratio for the high outcome matters. Then the no-excuse contract is strictly optimal.

We are going to show now that $\Gamma(\alpha)$ is either always increasing, or first increasing then decreasing.

$$
\Gamma^{\prime}(\alpha)=-\frac{1}{\alpha^{2}}\left(u_{H}^{\epsilon} / u_{L}\right)^{1 / \alpha}\left[\varphi(e) \ln \left(u_{H}^{\epsilon} / u_{L}\right)-\pi(e) \ln \left(u_{H} / u_{L}\right)\left(u_{H} / u_{H}^{\epsilon}\right)^{1 / \alpha}\right]
$$

Denote $\Phi$ the function in bracket. When $\alpha$ goes to zero, $\Phi$ goes to $-\infty$ because $u_{H} / u_{H}^{\epsilon}>1, \Phi$ is thus strictly negative. Moreover $\Phi$ is always increasing. Two cases are possible: $\Phi$ ends up to be still negative when $\alpha=$ 1 , then $\Gamma^{\prime}$ is always positive and $\Gamma$ always increasing. Or $\Phi$ becomes positive at some point and remains positive thereafter, because it is monotonic, then $\Gamma^{\prime}$ is first positive then negative and $\Gamma$ first increasing then decreasing.

To sum up, $\Gamma(\alpha)$ is strictly negative when $\alpha$ is close to zero and strictly positive for $\alpha=1$. In between this function is either always increasing, or increasing then decreasing. In both cases, by the intermediate value theorem, there exists one and only one $\left.\alpha_{2} \in\right] 0,1\left[\right.$ such that $\Gamma\left(\alpha_{2}\right)=0$. Moreover $\Gamma(\alpha)>0$ if and only if $\alpha>\alpha_{2}$ that is, the no-excuse contract is optimal if and only if $\alpha>\alpha_{2}$.

\section{Proof of Proposition 7}

When both limited liability constraints bind, the excuse contract is optimal if and only if

$$
\varphi(e) \frac{C}{\varphi(e)-\varphi(0)} \leq \pi(e) \frac{C}{\pi(e)-\pi(0)}
$$

This inequality holds if and only if $\varphi(e) / \varphi(0) \geq \pi(e) / \pi(0)$, that is, given Lemma 2, if and only if $R^{\epsilon} \geq R^{H}$. 


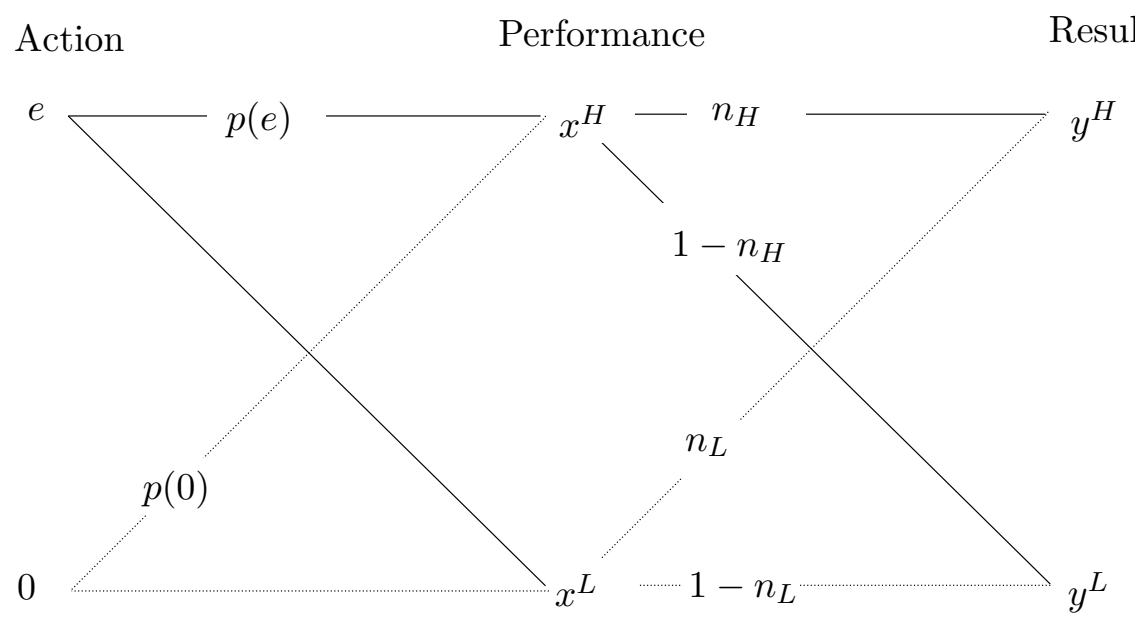

Figure 1: Information structure 


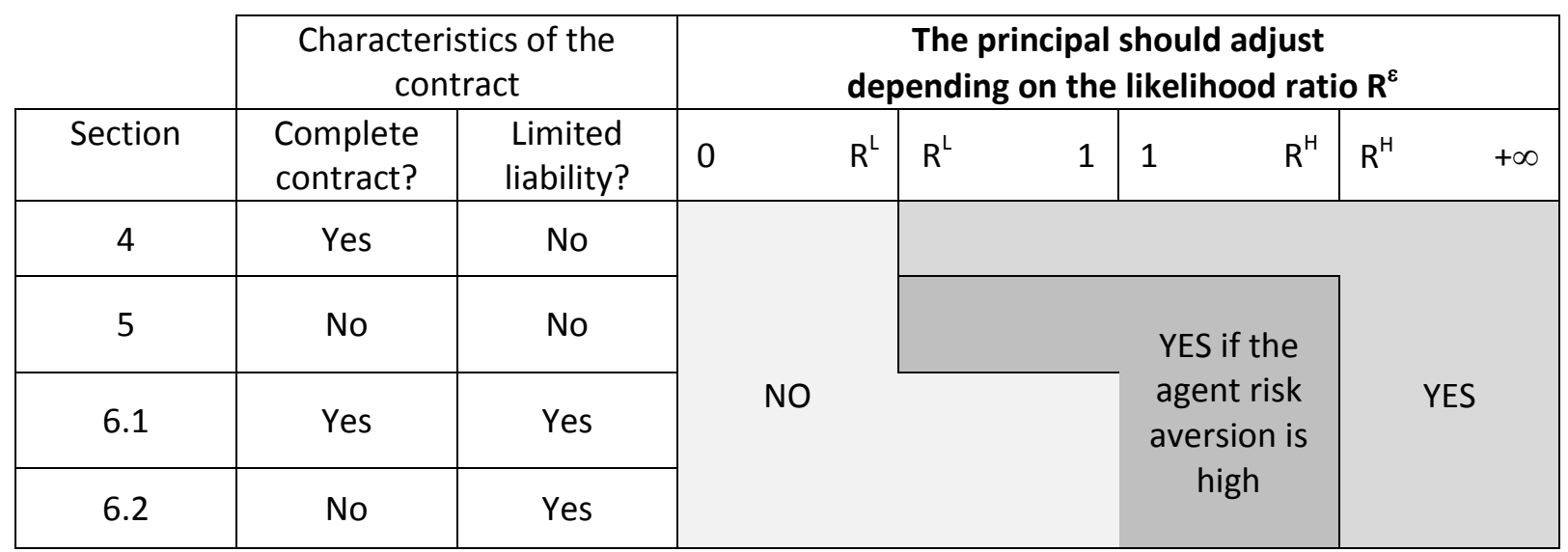

Figure 2. Summary of the results 